\title{
Stroke: a Hidden Danger of Margin Trading in Stock Markets
}

\author{
Shu-Hui Lin, Chien-Ho Wang, Tsai-Ching Liu, \\ and Chin-Shyan Chen
}

\begin{abstract}
Using 10-year population data from 2000 through 2009 in Taiwan, this is the first paper to analyze the relationship between margin trading in stock markets and stroke hospitalizations. The results show that 3 and 6 days after an increase of margin trading in the Taiwan stock markets are associated with greater stoke hospitalizations. In general, a $1 \%$ increase in total margin trading positions is associated with an increment of 2.5 in the total number of stroke hospitalizations, where the mean number of hospital admissions is 233 cases a day. We further examine the effects of margin trading by gender and age groups and find that the effects of margin trading are significant for males and those who are 45-74 years old only. In summary, buying stocks with money you do not have is quite risky, especially if the prices of those stocks fall past a certain level or if there is a sudden and severe drop in the stock market. There is also a hidden danger to one's health from margin trading. A person should be cautious before conducting margin trading, because while it can be quite profitable, danger always lurks just around the corner.
\end{abstract}

KEYWORDS Margin trading, Stroke, Stock market, Health, Taiwan

\section{INTRODUCTION}

Stock markets are one of the more important intermediate institutions that can help increase wealth. While investors may obtain a sizeable fortune from stock markets over a relatively short period, their actions in stock markets are invariably associated with high risk. In terms of financial theories, holding on to a risky asset is regarded as being more costly than holding on to a safe asset, essentially because the return of a risky asset may well be negative. However, aside from the financial cost of holding risky stocks, there may be other costs that cannot be measured in purely monetary terms, since the pursuit of gains in such a way may also be costly to the health of individual investors. When investors conduct arbitrary strategies in stock markets, the possibility of incurring a loss may cause huge mental and psychological stress for them. Bearing such pressures in the long run may actually give rise to long-lasting harm to one's health.

Lin is with the Department of Finance, National Changhua University of Education, 2, Shi-Da Road, Changhua City, 500, Taiwan; Wang and Chen are with the Department of Economics, National Taipei University, 151, University Rd., San Shia, New Taipei City, 23741, Taiwan; Liu is with the Department of Public Finance, National Taipei University, 151, University Rd., San Shia, New Taipei City, 23741, Taiwan.

Correspondence: Chin-Shyan Chen, Department of Economics, National Taipei University, 151, University Rd., San Shia, New Taipei City, 23741, Taiwan. (E-mail: stan@mail.ntpu.edu.tw) 
To help facilitate investors to trade over the short term, stock markets have set up many trading regulations. One common strategy is to borrow money from brokers to buy stocks, which is called margin trading. The most attractive feature of margin trading is that investors may use financial leverage to accumulate stocks whose aggregate buy-in value is greater than the cash a person had before purchasing them. Even though investors may amplify their profits by margin trading, it is a high-risk strategy that can also yield huge losses if executed incorrectly. Those with margin trading strategies invest under greater pressure, which may raise the incidences of strokes.

A rich strand of the literature is already available on the relationship between wealth and incidences of strokes. Brenner found a negative correlation between economic condition and mortality risk. ${ }^{1}$ However, some recent studies like Ruhm and Gerdtham and Ruhm, ${ }^{2,3}$ noted a positive correlation between economic conditions and mortality risk. Other studies have focused on the relationship between stock market index and personal health status. Fiuzat et al. examined the relationship between cardiovascular events as measured by the acute myocardial infarction (AMI) rate and the 2008 financial crisis in the USA, showing that the AMI rate increased when the stock market index fell. ${ }^{4} \mathrm{Ma}$ et al. explored the relationship between fluctuations of the Shanghai stock exchange composite index and coronary heart disease mortality, noting that the effect of stock index fluctuations on such mortality is robust. ${ }^{5}$ Schwartz et al. investigated the relationship between the 2008 stock market crash and cardiac deaths in Los Angeles County and found that the crash did not affect cardiac rates there after controlling seasonal variations. ${ }^{6}$ Lin, Chen, and Liu examined the impact of changes in the Taiwan stock weighted index on the nationwide prevalence of mental disorders, observing that stock price fluctuations clearly do affect the hospitalization of mental disorders. ${ }^{7}$ Chen et al. used population-based aggregate data to investigate the impact of stock market movements on incidences of strokes in Taiwan and noted that both a low stock index level and a daily fall in the stock index are associated with greater incidences of strokes. ${ }^{8}$ Although many studies have investigated the relationships between economic conditions and health outcomes, none have looked at the relationship between margin trading and personal health conditions.

Strokes are one of the leading causes of death in developed countries and indeed were the third major cause of deaths in Taiwan in 2009. A number of studies are now suggesting that strokes are set to become the second leading cause of death worldwide as well as one of the leading causes of disability. ${ }^{9-12}$ Incidences of strokes place an enormous burden on a country's national health system, mainly as a result of the requirement for ongoing care. $^{12-14}$ This issue has therefore attracted enormous attention, with a considerable number of studies investigating the risk factors associated with strokes, such as investment activities, urbanization, and socioeconomic status. ${ }^{8,15-18}$

The primary aim of this study is to examine the impact of risky investment strategies on the nationwide prevalence of strokes in Taiwan. While investors employing margin trading strategies may earn more wealth in a short period of time, they are also likely bearing a huge amount of stress. Falling stock markets may represent a substantial psychological stress that could adversely affect hospital admissions for strokes. To explore whether the way people manage their wealth affects the risk of stroke occurrence, we analyze the relationship between the change in margin trading levels and hospitalizations for strokes. The variation in margin trading is used as a proxy for changes in the extent of executing a risky financial investment strategy. In contrast to prior works that have analyzed strokes, the present study extends the current literature in two ways. First, we use population-based admissions at the aggregate level as our health measure. Second, margin trading data from the Taiwan stock market, which we use to measure the effect of risky investment strategies, extend earlier 
research papers that only concentrate on stock index movements. ${ }^{8}$ We investigate whether hospitalizations of cerebrovascular disease increase when investors choose high-risk investment strategies.

\section{METHODS}

We obtain data from the Taiwan National Health Insurance Research Database (NHIRD) and Taiwan Stock Exchange (TWSE) to examine the possible relationship between hospitalizations for strokes and the margin trading of stocks. Because the datasets used in the present study consist of de-identified secondary data released to the public, this study is exempt from a full review by the institutional review board. Our data cover the period from 1 January 2000 to 31 December 2009.

The dependent variable of interest to this study is the number of daily hospitalizations for strokes, with each hospitalization a result of a cerebrovascular attack (CVA, ICD-9 code 430-437) being counted as one stroke incidence. Taiwan initiated the National Health Insurance (NHI) program in 1995, and 99.5\% (about 22,928,190) of its citizens were enrolled in the program by the end of 2008 . The data on strokes derived from the Taiwan NHIRD are NHI hospitalization records, which are population-based and have an essential merit of avoiding the sampling bias issue. Our study comprises a total of 3653 observations on daily hospitalizations caused by CVA, with the mean number of CVA approximately 233 cases a day. The maximum and minimum numbers of daily CVA hospitalizations are 372 and 3 , respectively (Table 1).

The key independent variable is the percentage change in daily total margin trading positions. When investors buy stocks on margin, the margin trading position increases; conversely, the position decreases as investors liquidate those stocks bought on margin. We use the percentage change in total margin trading positions to measure the net increase (or decrease) amount that investors borrow to purchase stocks. For non-transaction days such as weekends and holidays, the percentage change value is set to zero. During our study period, the daily percentage change in total margin trading positions is $-0.012 \%$ on

TABLE 1 Summary statistics for variables

\begin{tabular}{lccccccr}
\hline Variable & Min. & Q1 & Median & Mean & Q3 & Max. & Std Dev \\
\hline Stroke & 3 & 205 & 239 & 233.724 & 263 & 372 & 45.411 \\
Stroke_M & 0 & 117 & 139 & 136.133 & 155 & 227 & 27.598 \\
Stroke_F & 3 & 84 & 98 & 97.425 & 111 & 164 & 20.395 \\
Stroke_2544 & 0 & 11 & 14 & 14.232 & 17 & 33 & 4.572 \\
Stroke_4564 & 3 & 62 & 72 & 71.662 & 82 & 128 & 14.989 \\
Stroke_6574 & 0 & 57 & 68 & 68.715 & 80 & 126 & 16.680 \\
Stroke_75 $_{\text {MarginChange }} t$ & -5.763 & -0.185 & 0 & -0.012 & 0.246 & 4.379 & 0.761 \\
Index $_{t}$ & 3.446 & 5.407 & 6.122 & 6.413 & 7.463 & 10.202 & 1.452 \\
Return $_{t}$ & -6.679 & -0.334 & 0 & 0.008 & 0.410 & 6.742 & 1.341 \\
SARS & 0 & 0 & 0 & 0.019 & 0 & 1 & 0.138 \\
\hline
\end{tabular}

Our data cover the period from 1 January 2000 to 31 December 2009 and consist of 3653 observations. Stroke is the daily number of hospitalizations owing to cerebrovascular attack (CVA, ICD-9 code 430-437). Stroke_M and Stroke_F are the daily number of CVA hospitalizations for male and female, respectively. Stroke_2544, Stroke_4564, Stroke_6574 and Stroke_75 are the daily number of CVA hospitalizations for four age groups. MarginChange $e_{t}$ is the percentage change in daily total margin trading position on date $t$. Index is the daily stock market index divided by 1000 . Return is the daily stock market index percentage change. For concise presentation, we omit the statistics of variables for SARS, months and weekdays 
average. The maximum percentage change is $4.38 \%$, while the minimum change is $-5.76 \%$, equating to about NT $\$ 11.5$ billion (US $\$ 349$ million, 1 US $\$=32.95$ NT $\$$ ) and -NT $\$ 15$ billion (-US\$455 million), respectively, in regard to the mean of total margin trading positions (NT $\$ 262$ billion, or US\$7.95 billion).

We employ SAS v 9.2 software for the statistical analyses performed in this study. The following autoregressive moving average with exogenous variable (ARMAX) regression examines the relationship between hospitalizations for strokes and the percentage change of total margin trading positions:

$$
\begin{aligned}
\text { Stroke }_{t}= & \alpha+\sum_{j=0}^{L} \beta_{1 j}\left(\text { MarginChange }_{t-j}+\beta_{2} \text { Index }_{t}+\beta_{3} \operatorname{Return}_{t}+\beta_{4} \operatorname{Return}_{t}{ }^{2}+\beta_{5} \text { SARS }_{t}\right. \\
& +\beta_{6} \operatorname{Trend}_{t}+\beta_{7} \operatorname{Trend}_{t}^{2}+\sum_{k=1}^{4} \rho_{k} \text { Stroke }_{t-k}+\sum_{i=1}^{6} \delta_{i} D i_{t}+\sum_{j=1}^{11} \gamma_{t} M j_{t}+\varepsilon_{t}
\end{aligned}
$$

Here, Stroke $e_{t}$ is the number of daily hospitalizations caused by strokes on date $t$. MarginChange $_{t-j}$ is the percentage change in daily total margin trading positions on date $t-\mathrm{j}$, with $j \geqq 0$. Investors who buy on margin usually target to hold the stock until its price rises, meaning the effect of margin trading persists at least until investors sell the stock and its impact on the incidences of strokes may have a time lag. When estimating the models, we consider a maximum lag of 7 MarginChange $_{t-j}$ to capture all potential effects of margin trading. Based on prior research results, we include stock market index $\left(\operatorname{Index}_{t}\right)$ and market return $\left(\right.$ Return $\left._{t}\right)$ variables in the models to control the impact of wealth variation in the short run on hospitalizations for strokes. ${ }^{8}$ Index $t$ is the daily closing level of the Taiwan Stock Exchange Capitalization-weighted Stock Index (TAIEX). Return ${ }_{t}$ is the percentage change in the daily closing level of TAIEX. For non-transaction days such as weekends and holidays, we set the value of Index $x_{t}$ equal to the previous transaction value and the value of Return $_{t}$ equal to zero.

We also include day-of-the-week dummies and month dummies to control for the tendency of strokes to occur on particular days and seasons. ${ }^{19-22} D i_{t}$ represents the dayof-the-week dummies with Monday as the reference day, and $M j_{t}$ notes the month dummies with December representing the reference month. The time-trend effect and SARS effect are both controlled in the models. ${ }^{8}$ The value of SARS is equal to one if the observation occurs during the SARS outbreak period (26 April 2003 to 5 July 2003); otherwise zero. Table 1 presents the summary statistics of the key variables.

The main benefit from the ARMAX regression model is that it, like Eq. (1), can analyze the post-margin trading effects on stroke hospital admissions. Some stroke patients might not have serious symptoms until 2-6 days after the values of their stock portfolio drop. If we only use OLS models, then the delayed effects on cerebrovascular hospital admissions cannot be detected. This study uses the time series ARMAX regression to explore the dynamic effects between stroke hospital admissions and the percentage of change in margin trading. Because the time series exhibit serial autocorrelations, we choose AR(4) based on Akaike Information Criterion (AIC) to control the possible serial correlations in residuals.

\section{RESULTS}

Table 2 displays the relationship between stroke hospitalizations and margin trading for the whole population. We consider contemporary and lag effects of margin 


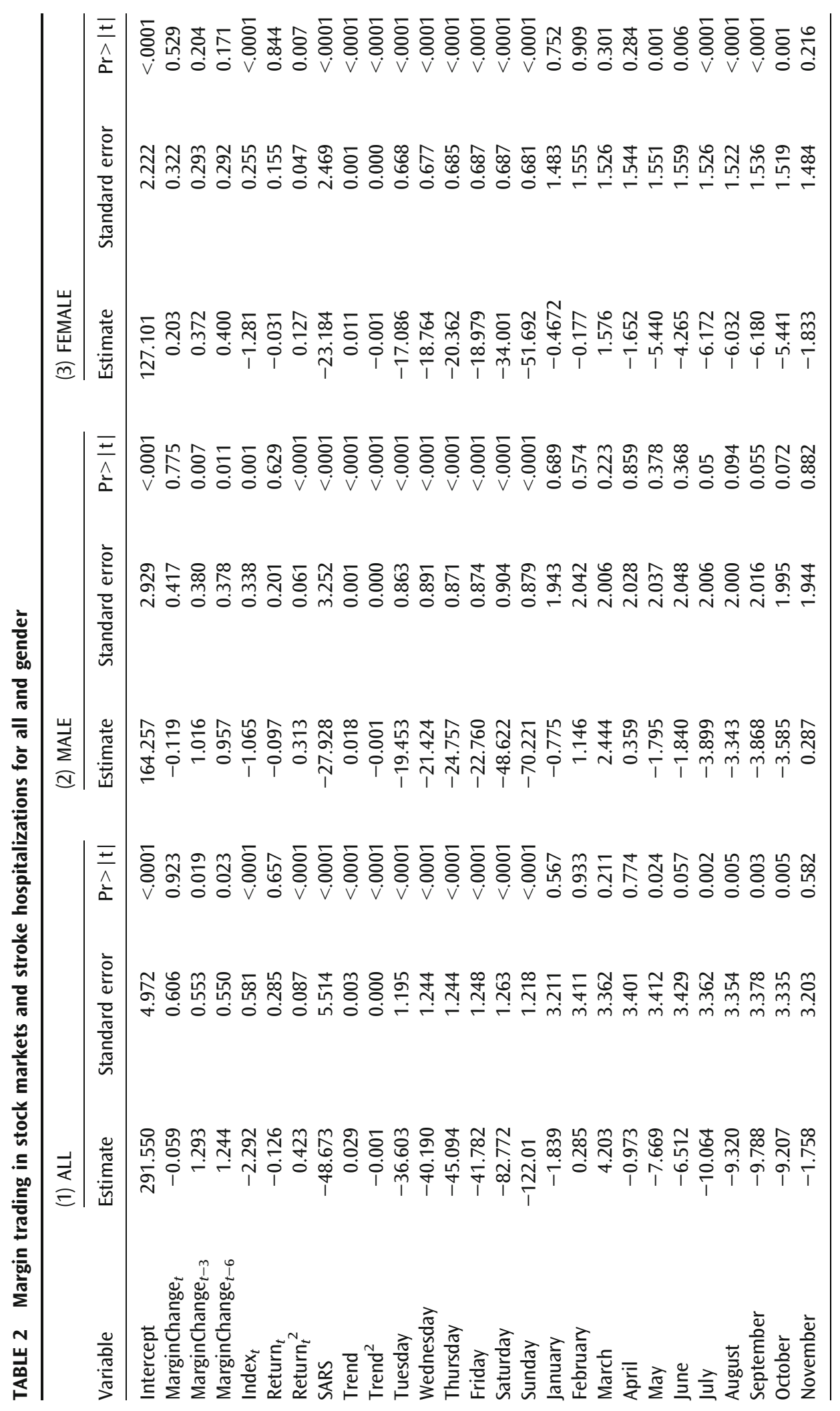




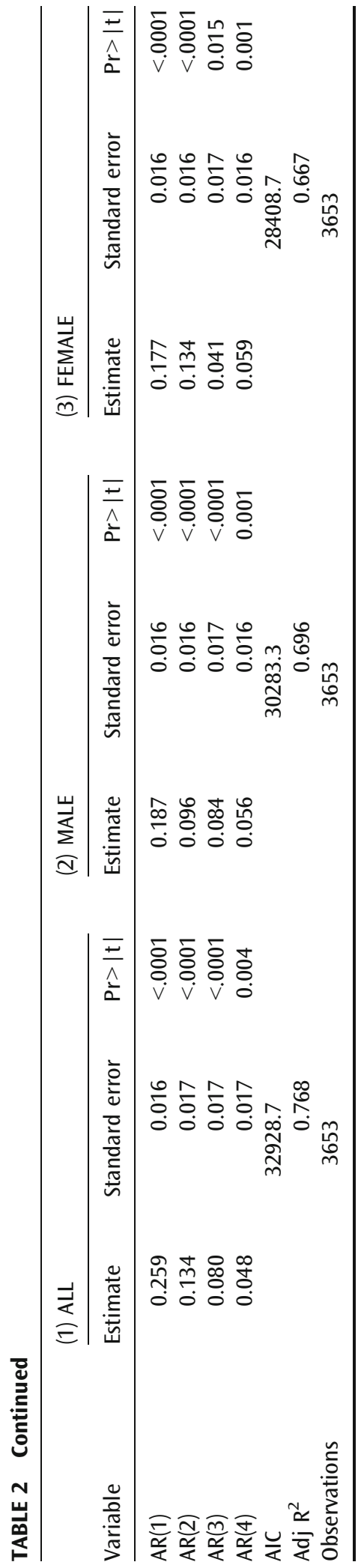


trading (MarginChange $e_{t-j}$ ) on these hospitalizations. We set the maximum lag number equal to $7(j=7)$ and investigate the lag effect of margin trading. The results indicate that previously buying stock on margin impacts the incidences of cerebrovascular disease, especially for trading on dates $t-3$ and $t-6$, whereas coincident margin trading does not have a prominent relationship with stroke hospitalizations. For brevity, Table 2 only presents the model with lag effects for dates $t-3$ and $t-6$, which have the most significant effect on the incidences of strokes. In regression 1 of Table 2, the coefficient of coincident margin trading $\left(\right.$ MarginChange $_{t}$ ) is negative but insignificant, suggesting that an increase in margin trading positions does not have a distinguishable influence on contemporary stroke incidences. The coefficients of MarginChange $t_{t-3}$ and MarginChange th $_{-6}$ are 1.3 and 1.2 , respectively. The positive and significant coefficients suggest that increasing stock purchases on margin leads to higher occurrences of stroke hospitalizations in the following days. In other words, 3 days after a $1 \%$ increase in total margin trading positions, daily stroke hospitalizations rise by 1.3 cases; 6 days after a $1 \%$ increase in total margin trading positions, daily stroke hospitalizations go up by 1.2 cases. In general, a $1 \%$ increase in total margin trading positions is associated with an increment of $2.5(=1.3+1.2)$ in the total number of stroke hospitalizations, where the mean number of hospital admissions is 233 cases a day. An alternative to the hospitalization data used in this paper is stroke outpatient data, but the nationwide outpatient dataset is not available to us. Chiu and Lien indicate that the amount of stroke outpatient utilization is about 13 times that of hospitalization utilization based on data from a medical center. ${ }^{23}$ The results of regression 1 in Table 2 also show that stroke hospitalizations have a significant relationship with stock market index level, market return, day of the week, and seasons, which are consistent with the findings of previous research.

We further examine the effects of margin trading by gender and age groups and present the results in regressions 2 and 3 of Table 2 . The results show that the effect of purchasing stock on margin is positive for both males and females, but significant only for males. For males, 3 days after a $1 \%$ rise in daily margin trading positions, daily stroke hospitalizations increase by 1.0 cases; 6 days after that $1 \%$ rise, daily stroke hospitalizations increase also by 1.0. In total, a $1 \%$ increase in margin trading positions results in a rise of $2.0(=1.0+1.0)$ in the number of stroke hospitalizations by males. Similar to the result of regression 1 , the contemporary daily change in margin trading positions does not have a significant effect on stroke hospitalizations for males.

The estimations of different age groups in Table 3 show that the daily change effects of margin trading are significant for people between 45 and 74 years old, but not for those younger than 45 and older than 74 . The significant coefficients indicate that a rise in margin trading positions leads to more stroke hospitalizations in the following days for people between 45 and 74 years old. For people between 45 and 64 years old, the margin trading effect is mainly from MarginChange $t_{t-6}$. In other words, 6 days after a $1 \%$ rise in daily margin trading positions, daily stroke hospitalizations increase by 0.6 cases for people between 45 and 64 years old. Furthermore, the impacts of MarginChange $e_{t-3}$ and MarginChange $_{t-6}$ are both significant for people between 65 and 74 years old. If margin trading positions move up $1 \%$, then by summing up the effects of MarginChange $t_{t-3}$ and MarginChange t $_{t-6}$, the number of stroke hospitalizations increases by $1.2(=0.6+0.6)$ cases for people between 65 and 74 years old. 


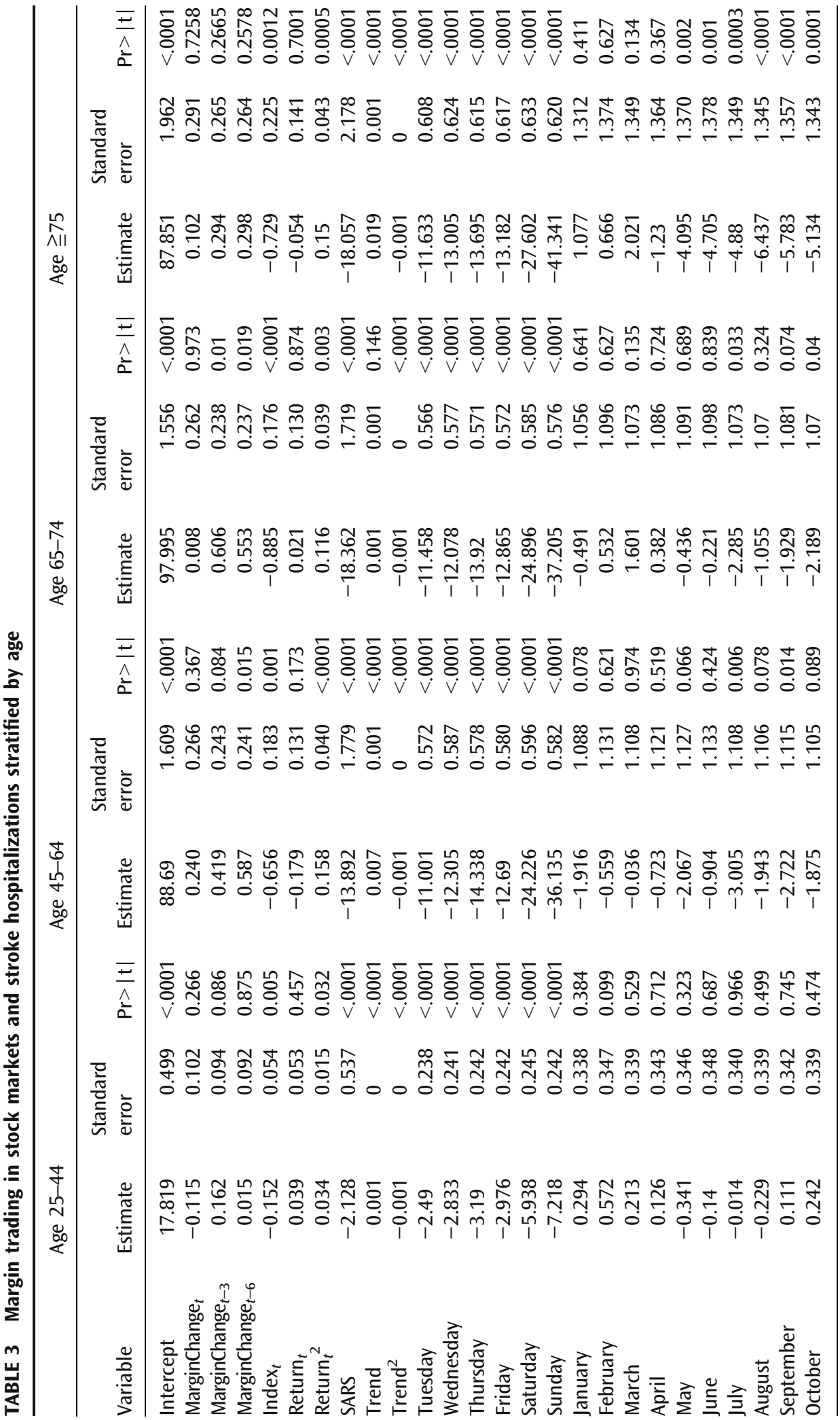




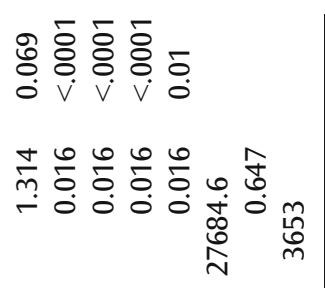

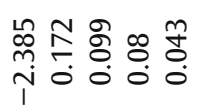

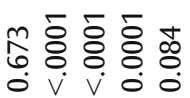

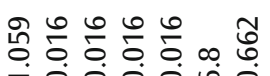

$\therefore$

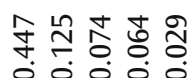

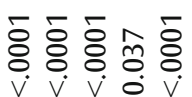

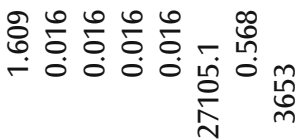

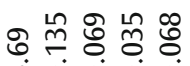

$\infty$ ○ 0.0

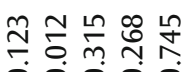

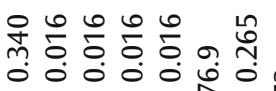

O०000

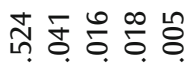

0000

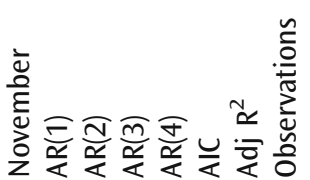




\section{DISCUSSION}

This study shows that leveraged investment is associated with an increase in stroke hospitalizations. Our analyses reveal that an increase in the daily change of margin trading positions leads to more stroke hospitalizations in the following days, especially the third and sixth days after the increase. One possible explanation for the results is that increasing one's margin trading can trigger a cerebrovascular attack, because margin trading is a high-risk investment strategy that amplifies both potential gains and losses. Since investors using margin trading strategies are trying to earn more wealth over a short period time, investors buying stocks on margin will be forced either to deposit more money into their account or to liquidate the stocks if the stock price decreases below a certain point. These features of margin trading easily induce psychological stress. Previous research has indicated that psychological conditions can influence a person's risk of cardiovascular diseases. ${ }^{24,25}$ Hamer et al. documented that psychological distress is connected with increased mortality from cerebrovascular disease. $^{25}$

One of the possible explanations for the lagging effect of margin trading may be due to regulation. In Taiwan, investors who receive a margin call do not need to sell off their stocks or add more cash to their margin account immediately; rather, they are forced to make this decision two business days after receiving the margin call. The two-day buffer time may lead them to become more stressed about this decision, which may produce a detrimental effect on their health. However, the buffer time for determining whether to liquidate the stock or add more cash into the account does not completely explain the lagging effects that take place on date $t-3$ and date $t-6$. There may be unobserved factors that occur later from the effect of margin trading.

We find that the effect of margin trading is significant for males and people between 45 and 74 years old, while it is not significant for females, for people above 74 , and for people below 45 . The potential reason for the significant effect on males is that they are more likely to initiate a margin trading strategy due to their attitude toward risk. Lab and field studies indicate that men are more risk-prone than are women, ${ }^{26}$ which may lead men to engage in risky investment. Researchers have also documented that males are more inclined to feel competent in money handling and willingly take risks to amass wealth. ${ }^{27}$ Given that men participate more actively in margin trading transactions than women, the impact of a daily change in margin trading positions is mainly influential on males.

Grable and Lytton indicate that risk tolerance decreases with age, ${ }^{28}$ implying that people over 74 years old are least likely to conduct margin trading activities, which might be the reason why the effect in the change of daily margin trading positions is insignificant for this age group. However, the insignificant effect upon people under 45 could be due to their physical health status, and not from their risk preference. People younger than 45 may have a healthier physical status, which can help ease any pressure induced by pursuing a risky investment strategy such as margin trading.

Our study does present some limitations. First, aggregated hospital admission data are used in our analysis. We have no information on whether those stroke hospitalization patients had engaged in margin trading in the stock market or not. We only find that there is a relationship between margin trading and stroke hospitalizations among different age and gender cohorts, but with the limitation of our aggregated admission data, we are unable to verify whether different age and gender cohorts have different preferences to engage in margin trading strategies. 
Future studies are needed to clarify the impacts of margin trading on stroke hospitalizations when richer data are available. Second, we are not able to control socioeconomic conditions of an individual while analyzing the relationship between margin trading and stroke hospitalizations. Socioeconomic conditions have been found to be important factors influencing one's personal health status. As such, the effects of margin trading on stroke hospitalizations may be overestimated if socioeconomic variables such as income and education are controlled.

\section{CONCLUSION}

Margin trading in stock markets is a high-risk strategy that can earn huge profits if an investor executes it properly. However, one can also lose a lot of money if the timing is wrong. The danger from margin trading in stock markets is not only financially but also physically. Using 10-year population data from 2000 through 2009 in Taiwan, this is the first paper to analyze the relationship between margin trading in stock markets and stroke hospitalizations. We find that 3 and 6 days after an increase of margin trading in the Taiwan stock market are associated with greater stroke hospitalizations. We further examine the effects of margin trading by gender and age groups and note that the effects of margin trading are only significant for males and those who are 45-74 years old. In summary, buying stocks with money you do not have (essentially borrowing money through margin trading) is quite risky, especially if the prices of those stocks fall past a certain level or if there is a sudden and severe drop in the stock market. There is also a hidden danger to one's health from margin trading. A person should be cautious before conducting margin trading, because while it can be quite profitable, danger always lurks just around the corner.

\section{ACKNOWLEDGMENTS}

This study is based in part on data from the National Health Insurance Research Database provided by the National Health Insurance Administration, Ministry of Health and Welfare and managed by National Health Research Institutes. The interpretation and conclusions contained herein do not represent those of National Health Insurance Administration, Ministry of Health and Welfare or National Health Research Institutes."

Funding Sources. This work is partly supported by the Ministry of Science and Technology in Taiwan through grant NSC 101-2410-H-305-054-MY3.Compliance with Ethical Standards. The data set used in this study consists of de-identified secondary data released to the public for research purpose. No ethics problem.

\section{REFERENCES}

1. Brenner M. Mortality and the national economy. Lancent. 1979; 314: 568-573.

2. Gerdtham U, Ruhm C. Deaths rise in good economic times: evidence from the OECD. Econ Hum Biol. 2006; 4: 298-316.

3. Ruhm C. Good time make you sick. J Health Econ. 2003; 22: 637-658.

4. Fiuzat P, Shaw L, Thomas L, Felker M and O'Connor C. United States stock market performance and acute myocardial infarction rates in 2008-2009 (from the Duke Databank for cardiovascular disease). Am J Cardiol. 2010; 106: 1545-1549. 
5. Ma W, Chen H, Jiang L, Song G, Kan H. Stock volatility as a risk factor for coronary heart disease death. Eur Heart J. 2011; 32: 1006-1011.

6. Schwartz B, Pezzullo J, McDonald S, Poole W and Kloner R. How the 2008 stock market crash and seasons affect total and cardic deaths in Los Angeles county. Am J Cardiol. 2012; 109: 1445-1448.

7. Lin C, Chen C, Liu T. Do stock prices drive people crazy? Health Policy Plan. 2015; 30: 306-314.

8. Chen C, Chen C, Liu T, Lin Y. Stock or stroke? Stock market movement and stroke incidence in Taiwan. Soc Sci Med. 2012; 75: 1974-1980.

9. Berger K, Schulte H, Stogbauer F, Assmann G. Incidence and risk factors for stroke in an occupational cohort: the PROCAM Study. Stroke. 1998; 29: 1562-1566.

10. Cox A, McKevitt M, Rudd A, Wolfe C. Socioeconomic status and stroke. Lancet Neurol. 2006; 5: 181-188.

11. van Rossum C, van de Mheen H, Breteler M, Grobbee D, Mackenbach J. Socioeconomic differences in stroke among Dutch elderly women: the Rotterdam Study. Stroke. 1999; 30: 357-362.

12. Wolf P, D'Agostino R, Belanger A, Kannel W. Probability of stroke: a risk profile from the Framingham Study. Stroke. 1991; 22: 312-318.

13. Jakovljevic D, Sarti C, Sivenius J, et al. Socioeconomic status and ischemic stroke: the FINMONICA stroke register. Stroke. 2001; 32: 1492-1498.

14. Saka O, McGuire A, Wolfe C. Cost of stroke in the United Kingdom. Age Aging. 2009; 38: 27-32.

15. Brown P, Guy M, Broad J. Individual socio-economic status, community socio-economic status and stroke in New Zealand: a case control study. Soc Sci Med. 2005; 61: 11741188.

16. Diez-Roux A, Link B, Northridge M. A multilevel analysis of income inequality and cardiovascular disease risk factors. Soc Sci Med. 2000; 50: 673-687.

17. Hart C, Hole D, Smith G. The contribution of risk factors to stroke differentials, by socioeconomic position in adulthood: the Renfrew/Paisley Study. Am J Public Health. 2000; 90: 1788-1791.

18. Lin H, Lin Y, Liu T, Chen C, Chiu W. Urbanization and stroke prevalence in Taiwan: analysis of a nationwide survey. J Urban Health. 2007; 84: 604-614.

19. Chen Z, Chang S, Su C. Weather and stroke in a subtropical area: Ilan, Taiwan. Stroke. 1995; 26: 569-572.

20. Jakovljevic D. Day of the week and ischemic stroke: is it Monday high or Sunday low? Stroke. 2004; 35: 2089-2093.

21. Kelly-Hayes M, Wolf P, Kase C, Brand F, McGuirk J, D’Agostino R. Temporal patterns of stroke onset: the Framingham Study. Stroke. 1995; 26: 1343-1347.

22. Ricci S, Celani M, Vitali R, la Rosa F, Righetti E, Duca E. Diurnal and seasonal variations in the occurrence of stroke: a community-based study. Neuroepidemiology. 1992; 11: 5964.

23. Chiu H, Lien L. Stroke analysis in Shin-Kong hospital (in Chinese). Acta Neurol Taiwan. 2003; 12: 284-288.

24. Brotman D, Golden S, Wittstein I. The cardiovascular toll of stress. Lancet. 2007; 370: 1089-1100.

25. Hamer M, Kivimaki M, Stamatakis E, Batty D. Psychological distress as a risk factor for death from cerebrovascular disease. Can Med Assoc J. 2012; 184: 1461-1466.

26. Croson R, Gneezy U. Gender differences in preferences. J Econ Lit. 2009; 47: 448-474.

27. Prince M. Women, men, and money styles. J Econ Psychol. 1993; 14: 175-182.

28. Grable J, Lytton R. Investor risk tolerance: testing the efficacy of demographics as differentiating and classifying factors. Financial Couns Plan. 1998; 9: 61-73. 